\title{
Minioptical Navigation System for CT-Guided Percutaneous Liver Procedures
}

\author{
David A. Valenti ${ }^{1}$, Louis-Martin Boucher ${ }^{1}$, Giovanni Artho ${ }^{1}$, \\ Christopher von Jako $^{2}$, Tatiana Cabrera ${ }^{1}$ \\ ${ }^{1}$ Department of Medical Imaging, McGill University, Montreal, Canada \\ ${ }^{2}$ ActiViews, Inc., Wakefield, USA \\ Email: david.valenti@mcgill.ca
}

Received May 3, 2013; revised June 7, 2013; accepted July 2, 2013

Copyright (C) 2013 David A. Valenti et al. This is an open access article distributed under the Creative Commons Attribution License, which permits unrestricted use, distribution, and reproduction in any medium, provided the original work is properly cited.

\begin{abstract}
Purpose: To evaluate a new miniature optical navigation system for CT-guided liver interventions. Material and Methods: A two-center, prospective study was performed with four interventional radiologists. A total of 20 patients had CT-guided liver biopsy or ablation interventions utilizing the CT-Guide ${ }^{\circledR}$ navigation system (ActiViews Inc., Wakefield, MA) between July 2011 and December 2011. The navigation system consists of a self-adhesive patientsticker printed with coincident colored and radio-opaque reference markers, a miniature disposable video camera that clips on and off an interventional instrument, and software loaded on a computer to display the navigation information. The primary end point was the frequency of a satisfactory instrument position for the intended intervention. Results: The cohort consisted of 13 males and 7 females with an average age of 63.1 years (range of 38 to 80). Most of the patients, 70\%, underwent CT-guided liver biopsy while the remainder had CT-guided ablation therapy. The average lesion size was $3.1 \mathrm{~cm}$ (range of $1.1-6.9 \mathrm{~cm}$ ). All of the interventions, regardless of lesion size, met the primary end point of satisfactory instrument positioning. There were no device-related or unexpected adverse events recorded. Only one patient had a mild adverse event and it resolved without intervention. Conclusions: This study demonstrated the safety and effectiveness of the CT-Guide ${ }^{\circledR}$ navigation system for CT-guided liver interventions, for both biopsies and ablations. The targeting success rate for a satisfactory intervention was $100 \%$ with the system.
\end{abstract}

Keywords: Computerized Navigation; CT-Guided Biopsy; CT-Guided Ablations

\section{Introduction}

The use of CT-guidance for hepatic interventions has become an accepted standard of care for diagnostic biopsies and therapeutic ablations where ultrasound guidance does not adequately visualize the lesion and there is a difficult and narrow safe instrument tract to the lesion. CT-guidance for liver biopsies has been reported to have an overall accuracy of $97 \%$, with sensitivity rates of $92 \%$ and specificity rates of $96 \%$ [1]. Radiofrequency ablations (RFA) for primary or secondary malignancies can be curative for selected patients [2], but the therapeutic efficacy is dependent on accurate instrument placement. Major complication rates are low and procedure-related mortalities are rare for both biopsy [3] and RFA procedures [4-6].

The effective use of CT-guidance is highly dependent on the skill and experience of the radiologist and may require a number of trial and error cycles in which re- peated CT scans are performed, especially when the target is small or deep, or the optimal path to the target is at a difficult angle. In order to meet these challenges CT fluoroscopy was developed by Katada et al. in 1993 to allow real-time CT guidance [7]. However, although various techniques for $\mathrm{CT}$-fluoroscopic guidance are used to reduce patient and physicians radiation dose to acceptably low levels [8], this reduction can be challenging to obtain, especially in cases where there is a steep angle to the target of interest. In view of this and especially of recent increased public sensitivity to medical radiation, many physicians have lost the initial enthusiasm that had built up for this method.

The basis of image-guided surgery and integrated imaging systems for intraoperative surgical planning was described in 1985 [9], and in the past 20 years a number of surgical navigation systems have been commercialized for different specialties. Surgical navigation has enabled physicians to locate and track the path of interventional 
instruments in relation to diagnostic images, which is analogous to the way a GPS (global positioning system) device locates a car using stored roadmaps. Conventional surgical navigation configurations utilize either optical or electromagnetic (EM) tracking technology to register the spatial location of a tracked instrument $[10,11]$.

The purpose of this study is to evaluate, in a clinical setting, the effectiveness and safety of a new navigation device that utilizes a miniature optical tracking technology to facilitate CT-guided instrument placement for liver interventions. This was performed in a prospective manner at two different centers with four radiologists.

\section{Materials and Methods}

\subsection{Study Design and Patient Data}

This was a prospective, single-arm study at two centers in Canada. Four staff radiologists with experience in CTguided interventional procedures participated in the study where the procedures were conducted between July 2011 and December 2011. The study protocol was designed by one of the participating radiologists in collaboration with the device manufacturer and subsequently approved by the Research Ethics Boards (REB) at each of the participating centers prior to patient enrollment.

Each radiologist participating in the study was required to attend a training session on the use of the navigation device and use it clinically at least three times before enrolling patients in the study. A principal investtigator was designated at each center, with responsibility for compliance with the study protocol and for reporting any deviation to the REB. The study was sponsored by the device manufacturer (ActiViews, Inc., Wakefield, MA) and monitored by an independent contract research organization. The study was conducted in accordance with the standard operating procedures of the each center's REB and the contract research organization. All data were prospectively collected in detailed case report forms by radiology research coordinators at each center under the supervision of the principal investigators.

All patients clinically indicated for CT-guided liver biopsy or ablation procedure at one of the participating centers were screened for compliance with the study inclusion and exclusion criteria. The inclusion criteria were: patients aged between 18 to 80 years old at the time of enrollment, ability to comply with the requirements of the study procedures, and a verified home address and phone number to facilitate study follow-up. The criteria for exclusion from enrollment were: patients with significant coagulopathy that cannot be adequately corrected, patients who participated in an active stage of any drug, intervention, or treatment trial within 30 days of enrollment, patients with preexisting conditions, which, in the opinion of the investigator, interfere with the con- duct of the study, patients who are uncooperative or cannot follow instructions, patients with a mental state that may preclude completion of the study procedure, and female patients who are pregnant or nursing. Written informed consent was obtained from all patients prior to the procedure. Patient data collection on the day of the procedure included demographics, relevant medical history, relevant concomitant medications, and vital signs.

Targeting accuracy was the primary efficacy endpoint and was defined by the ability to place the instrument at a location suitable for the planned intervention. The investtigator carefully reviewed the final instrument position on the post-placement CT images to determine this. This information was used to calculate success rate. In addition, secondary end points included: 1) the total time of the procedure(time between the first CT scan for selecting the skin entry point and the last $\mathrm{CT}$ scan performed following removal of the interventional instrument used), 2) the navigation time (the time between the calibration of instrument length and the CT scan showing the instrument in place for the intervention), 3) total number of CT scans performed throughout the procedure, and 4) the subject's radiation exposure during the procedure.

Safety was documented by evaluating all the adverse events (AEs), as well as thorough the assessment of AEs commonly associated with liver biopsies and ablations (e.g., severe pain, hemorrhage, unintended organ injury, pneumothorax, infection, etc.).

Vital signs were recorded once before the procedure, during three hours of post-procedure monitoring, and before subject discharge. AEs were collected and recorded following the completion of the procedure, during the observation time, and before discharge. The subjects were also followed-up with a phone call at 14 days \pm 5 days post procedure to assess AEs related to the procedure. A subject's enrollment in the study was completed after the follow-up phone call and after resolution of any procedure related $\mathrm{AE}$.

The primary endpoint results are reported with associated confidence interval (CI) and the secondary endpoints are reported with associated mean, maximum, minimum, and median values. All AEs are presented by their frequency and severity. The safety assessments are based on AEs reported by the subject or observed by the investigator. All AEs within the study duration were recorded and assessed with information regarding onset, duration (start and end times/dates), frequency, severity, seriousness, relationship to the navigation device, action taken, and outcome.

We invited 20 consecutive patients who met the criteria to participate in the study. There were two patients that signed the informed consent, but were not ultimately enrolled due to an alteration in their treatment unrelated to the guidance system, which was made by the investi- 
gator. These patients were replaced to maintain the sample size. The 20 patients that were enrolled had an average age of $63.1 \pm 10.6$ (range of 38 to 80 ) years, and the cohort consisted of 13 males $(65 \%)$ and 7 females $(35 \%)$.

To ensure diversity in the study population, a minimum number of cases with special infrequent conditions were required. These special requirements could be counted in more than one patient, and the groups were as follows:

- Group 1: at least 5 cases of lesion size equal or less than $2.0 \mathrm{~cm}$ in diameter.

- Group 2: at least 2 cases of intervention in the left lobe.

- Group 3: at least 2 cases of lesions deeper than $8 \mathrm{~cm}$ from the skin.

- Group 4: at least 3 cases of decubitus subject position.

\subsection{Navigation System}

CT-Guide ${ }^{\circledR}$ navigation (ActiViews Inc., Wakefield, MA) is a novel variant of traditional optically-based tracking systems that employs three main components: 1) a sterile, self-adhesive, registration sticker with coincident video and radiological x-ray imaging visible reference markers (Figure 1(a)); 2) a miniature disposable video camera that is easily clipped onto or off any standard interventional instrument (Figure 1(b)); and 3) proprietary threedimensional (3D) navigation software displayed on a large flat panel monitor and loaded into a computer that is housed by a mobile workstation (Figure 1(c)) inside the CT suite.

The registration sticker is attached to a patient prior to CT imaging. The sticker contains eight coincident reference markers that are visible on both a video image and the CT image. Printed on the front side of the sticker are eight color-coded markers, and the backside has eight coincident radio-opaque markers. The sticker also contains three discrete "entry holes" with a diameter suitable for the instrument gauge used in the intervention.
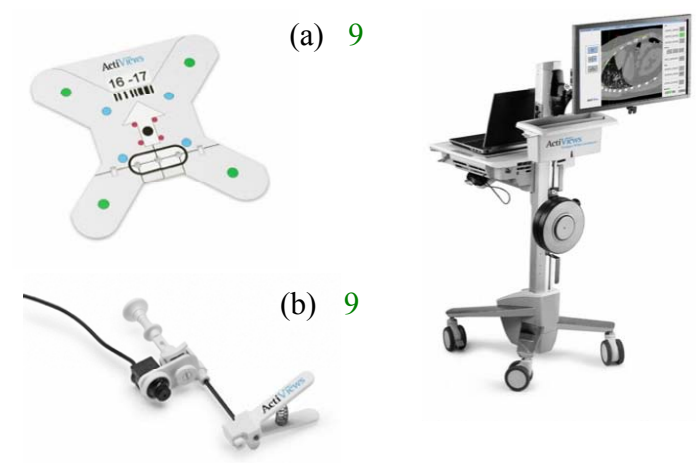

(c) 9

Figure 1. Navigation system comprises of a patient registration sticker (a), a miniature video camera that mounts on an instrument (b), and 3D software that is viewed on a flat panel screen (c).
During intervention, the miniature video camera is clipped onto the proximal end of an instrument, and provides a real-time video stream of the colored markers to the computer (Figure 2). Based on theses images, the navigation software enables virtual tracking of the instrument in the 3D CT image space relative to the sticker while being advanced toward a user-selected anatomical target.

\subsection{Procedure Technique}

The 20 patients were admitted to a dedicated day unit where the study was explained and consent obtained by a research study coordinator. The radiologist performing the procedure obtained a separate consent for the CTguided biopsy or ablation procedure. The patients undergoing a liver biopsy were given a local anesthetic with mild conscious sedation, if needed, while the ablation subjects were all under general anesthesia. The interventions were performed at both centers using a 64-slice Discovery CT750 HD scanner (GE Healthcare, Waukesha, WI). All CT scans were performed using a short helical acquisition tailored to the lesion location. Scout views were taken to locate the liver lesion. CT images were acquired through the lesion with a localizing grid placed on the patient's skin with $2.5 \mathrm{~mm}$ thick slices and $1 \mathrm{~mm}$ spacing between slices. The CT images were then automatically sent to the navigation computer in the CT suite.

The navigation software facilitated selection of the optimal entry point and path using interactive $3 \mathrm{D}$ views of the anatomy along the planned instrument path. The selected entry point was then marked on the skin surface and the area cleaned and prepped in the usual sterile manner. The sterile sticker was placed on the skin with the middle entry hole placed over the marked entry point and local anesthesia was administered using 1\% lidocaine.

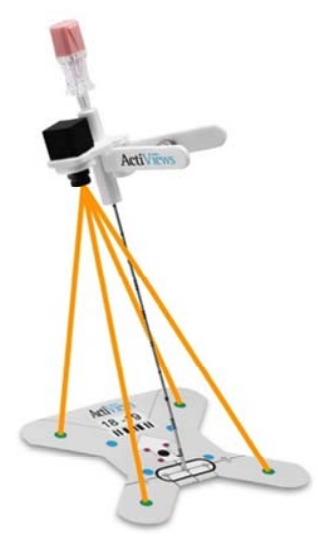

Figure 2. The miniature video camera is mounted on the instrument and captures the fidicuial markers that allows for instrument tracking in CT space. 
The sticker and lesion were then scanned with location information provided from the navigation system using thinner $1.5 \mathrm{~mm}$ slice thickness with $1 \mathrm{~mm}$ spacing between slices. The CT images were again automatically transmitted to the navigation computer. The reference markers were registered semi-automatically with a single-click and target location was confirmed on the newly acquired "sticker scan". The navigation software then displayed the instrument trajectory in three orthogonal planes and calculated the minimum instrument length required to reach the selected target.

A gauge-specific sterile miniature video camera was then clipped onto the instrument and a simple fast length calibration step was performed. The navigation information is displayed in three components: a graphic simulation of the instrument is overlaid on two orthogonal CT images with a display of the planned path from the entry point to the target and a video image from the camera is displayed with a graphic of the instrument tip position relative to the target with real-time feedback of the remaining distance between them. From the navigational information, the investigator first aligns the instrument's trajectory and advances it to the target (Figure 3). On reaching the pre-defined target, the miniature video camera is unclipped from the instrument and a confirmatory CT was obtained. Further instrument tip adjustments were made as necessary. Once the optimal instrument positioning was confirmed, the biopsy or ablation was performed as usual. A post procedural CT scan was performed after the instrument was removed in order to detect possible AEs.

\section{Results}

Of the 20 patients that completed the study, 14 biopsies (70\%) and 6 ablations (30\%) were performed. The average target lesion size in this cohort was $3.1 \pm 1.9 \mathrm{~cm}$ with a median of $35.0 \mathrm{~cm}$, a minimum size of $1.1 \mathrm{~cm}$ and a maximum of $6.9 \mathrm{~cm}$, and the average depth to target was

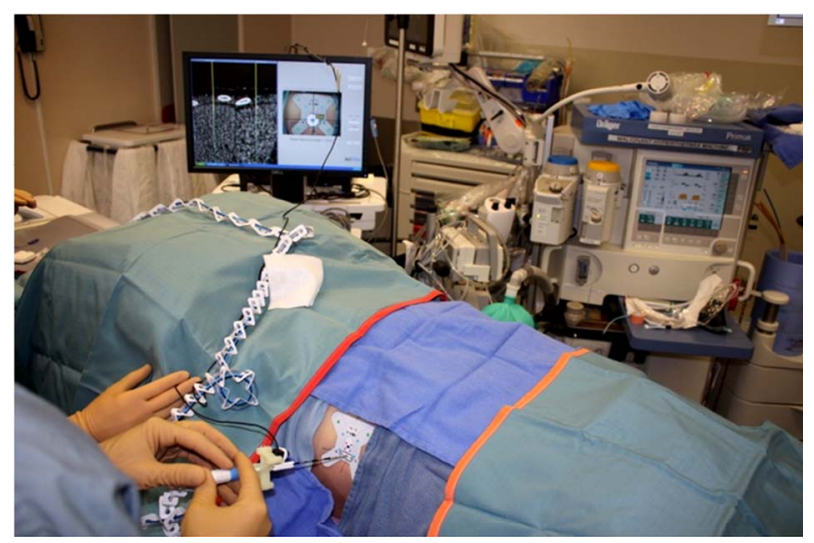

Figure 3. The user is advancing the ablation probe into the liver based on the navigation information.
$8.0 \pm 2.4 \mathrm{~cm}$ with a range from 3.4 to $12.7 \mathrm{~cm}$.

The success rate of the primary efficacy endpoint for the frequency of placing the instrument at a location suitable for the planned intervention, as determined by the investigator reviewing the CT images, was $100 \%$ with a 95\% CI. Table 1 presents the results of the secondary end points measured in the study, for the different special infrequent condition groups as well as for the total study cohort.

No device-related or unexpected AEs were recorded. Only one mild instance of pneumonitis was reported. This was in the first procedure and was an ablation procedure. This AE was not considered device related and was attributed to the extreme proximity of the ablated lesion to the pleural surface. The event was categorized as an unintended organ injury, which is a common AE in percutaneous liver procedures. It resolved without intervention or sequelae prior to discharge from the hospital.

\section{Discussion}

The CT-Guide ${ }^{\circledR}$ navigation was described previously in a laboratory study where it was shown to have an accuracy of less than $3 \mathrm{~mm}$ [12]. These results compared favorably to other navigation systems being used in CT-guided procedures [13-15]. However, these other reviewed navigation systems for CT-guided procedures utilize EM tracking technology where there can be significant limitations such as cost, system footprint, complexity, and environmental accuracy issues. The EM-based system accuracy degrades in the presence of metallic objects, which distort the magnetic field that is created for navigation $[11,16]$. These constraints of EM tracking technologies do not limit this technology, whereas the minioptical navigation system provided reliable accuracy in our environment and integrated with ease into our existing workflow.

At our institution, CT-guided liver procedures for biopsies and ablations are performed routinely in a conventional manner where check CT scans are performed during the intervention when the physician feels they are required. During each check CT scan, the staff exits the CT suite during the scan and the physician also evaluates the updated CT images in the control room. This method can be time consuming, but even more so when the target is small and in a challenging location that requires an offaxis trajectory. The potential use of navigation systems in CT-guided procedures is to guide the physician in the initial aiming of the instrument at the target and as a result reaching it quicker and more efficiently. While this study was not constructed to compare the time with and without navigation, the investigators did improve their total time with each case performed and observed the case time became more predicable without regard of the difficulty. 
Table 1. Detailed results of the patient study.

\begin{tabular}{|c|c|c|c|c|c|c|}
\hline & & $\begin{array}{l}\text { Group } 1(\mathrm{~N}=7) \\
(\text { lesion } \leq 2.0 \mathrm{~cm})\end{array}$ & $\begin{array}{l}\text { Group } 2(\mathrm{~N}=4) \\
\quad \text { (Left Lobe) }\end{array}$ & $\begin{array}{l}\text { Group } 3(\mathrm{~N}=11) \\
(\geq 8 \mathrm{~cm} \text { depth })\end{array}$ & $\begin{array}{c}\text { Group } 4(\mathrm{~N}=3) \\
\text { (Decubitus position) }\end{array}$ & Total $(\mathrm{N}=20)$ \\
\hline \multirow[t]{3}{*}{ Total Procedure Time (min) } & Mean (SD) & $49.3(20.5)$ & $35.0(5.7)$ & $49.3(18.6)$ & $57.7(16.6)$ & $42.6(18.2)$ \\
\hline & Median & 38.0 & 46.0 & 45.5 & 63.0 & 35.0 \\
\hline & Range & $25-57$ & $28-42$ & $31-82$ & $22-56$ & $23-82$ \\
\hline \multirow[t]{3}{*}{$\begin{array}{l}\text { Time Required To Guide The } \\
\text { Instrument To Target (min) }\end{array}$} & Mean (SD) & $5.3(2.0)$ & $5.7(2.5)$ & $4.6(1.9)$ & $5.0(2.1)$ & $3.9(2.0)$ \\
\hline & Median & 5.0 & 4.5 & 4.0 & 7.0 & 4.0 \\
\hline & Range & $2-8$ & $1-7$ & $2-8$ & $4-8$ & $1-8$ \\
\hline \multirow[t]{3}{*}{ Number of CT Scans } & Mean (SD) & $5.6(1.6)$ & $5.8(0.5)$ & $6.1(1.5)$ & $6.0(1.5)$ & $5.6(1.4)$ \\
\hline & Median & 7 & 6 & 6 & 7 & 5 \\
\hline & Range & $5-9$ & $5-6$ & $4-9$ & $5-8$ & $4-9$ \\
\hline \multirow[t]{3}{*}{$\begin{array}{c}\text { Radiation Exposure } \\
\text { Dose Length Product }\left(\mathrm{mGy}^{*} \mathrm{~cm}\right)\end{array}$} & Mean (SD) & $1750.1(847.7)$ & $1611.5(1158.7)$ & $1894.8(817.2)$ & $2128.0(1267.4)$ & $1778.8(855.2)$ \\
\hline & Median & 1600 & 1206 & 1608 & 1847 & 1543 \\
\hline & Range & $1389-3777$ & $760-3274$ & $965-3777$ & $1389-3777$ & $760-3777$ \\
\hline
\end{tabular}

*Navigation time is the time between the calibration of instrument length and the CT scan showing the instrument in place for the intervention.

Over the past 20 years, navigation systems have assisted physicians in a number of other specialties to overcome challenges related to percutaneous image-guided targeting. These techniques have been slow to adopt into interventional radiology applications mainly because of the lack of availability of a simple, accurate, and cost effective solution. This study demonstrated that CTGuide ${ }^{\circledR}$ navigation is simple, safe, and effective in guiding optimal instrument placement for liver interventions.

\section{REFERENCES}

[1] M. Lüning, B. Schmeisser, H. Wolff, W. Schöpke, E. Hoppe and R. Meyer, "Analysis of the Results of 96 CTGuided Fine Needle Biopsies of Liver Masses," Fortschr Röntgenstr, Vol. 141, No. 9, 1984, pp. 267-275. doi:10.1055/s-2008-1053132

[2] J. Bruix and M. Sherman, "Management of Hepatocellular Carcinoma," Hepatology, Vol. 42, No. 5, 2005, pp. 1208-1036. doi:10.1002/hep.20933

[3] K. R. Reddy and E. R. Schiff, "Gastrointestinal Emergencies," Williams \& Wilkins, Baltimore, 1996-1997.

[4] S. A. Curley, P. Marra, K. Beaty, L. M. Ellis, J. N. Vauthey, E. K. Abdalla, C. Scaife, C. Raut, R. Wolff, H. Choi, E. Loyer, P. Vallone, F. Fiore, F. Scordino, V. De Rosa, R. Orlando, S. Pignata, B. Daniele and F. Izzo, "Early and Late Complications after Radiofrequency Ablation of Malignant Liver Tumors in 608 Patients," Annals of Surgery, Vol. 239, No. 4, 2004, pp. 450-458. doi:10.1097/01.sla.0000118373.31781.f2

[5] T. de Baere,O. Risse, V. Kuoch, C. Dromain, C. Sengel, T. Smayra, M. Gamal El Din, C. Letoublon, D. Elias,
"Adverse Events during Radiofrequency Treatment of 582 Hepatic Tumors," American Journal of Roentgenology, Vol. 181, No. 3, 2003, pp. 695-700. doi:10.2214/ajr.181.3.1810695

[6] J. Kettenbach, W. Kostler, E. Rucklinger, B. Gustorff, M. Hüpfl, F. Wolf, K. Peer, M. Weigner, J. Lammer, W. Müller and S. N. Goldberg, "Percutaneous Saline-Enhanced Radiofrequency Ablation of Unresectable Hepatic Tumors: Initial Experience in 26 Patients," American Journal of Roentgenology, Vol. 180, No. 6, 2003, pp. 1537-1545. doi:10.2214/ajr.180.6.1801537

[7] K. Katada, R. Kato, H. Anno, Y. Ogura, S. Koga, Y. Ida, M. Sato and K. Nonomura, "Guidance with Real-Time CT Fluoroscopy: Early Clinical Experience,” Radiology, Vol. 200, No. 3, 1996, pp. 851-856.

[8] E. K. Paulson, D. H. Sheafor, D. S. Enterline, H. P. McAdams and T. T. Yoshizumi, "CT FluoroscopyGuided Interventional Procedures: Techniques and Radiation Dose to Radiologists," Radiology, Vol. 220, No. 1, 2001, pp. 161-167.

[9] G. Jako, "Lasers Cut a Swath in Surgical and Medical Applications," IEEE Spectrum, Vol. 22, 1985, pp. 82-87. doi:10.1109/MSPEC.1985.6370594

[10] R. Buchalla, S. Hopf-Jensen, O. Rubarth and W. Börm, "Frameless Navigated Biopsy with the BrainLAB ${ }^{\circledR}$ VarioGuide System: A Technical Note," Journal of Neurological Surgery. Part A, Central European Neurosurgery, 2012, in Press.

[11] R. A. von Jako, J. A. Carrino, K. S. Yonemura, G. A. Noda, W. Zhue, D. Blaskiewicz, M. Rajue, D. E. Groszmann and G. Weber, "Electromagnetic Navigation for Percutaneous Guide-Wire Insertion: Accuracy and Efficiency Compared to Conventional Fluoroscopic Guid- 
ance," Neuroimage, Vol. 47, Suppl. 2, 2009, pp. 127-132. doi:10.1016/j.neuroimage.2009.05.002

[12] C. von Jako, Y. Zuk, O. Zur and P. Gilboa, "A Novel Accurate Minioptical Tracking System for Percutaneous Needle Placement," IEEE Transactions on Biomedical Engineering, Vol. 60, No. 8, 2013, pp. 2222-2225. doi:10.1109/TBME.2013.2251883

[13] B. J. Wood, H. Zhang, A. Durrani, N. Glossop, S. Ranjan, D. Lindisch, E. Levy, F. Banovac, J. Borgert, S. Krueger, J. Kruecker, A. Viswanathan and K. Cleary, "Navigation with Electromagnetic Tracking for Interventional Radiology Procedures: A Feasibility Study," Journal of Vascular and Interventional Radiology, Vol. 16, No. 4, 2005, pp. 493-505. doi:10.1097/01.RVI.0000148827.62296.B4

[14] J. Krucker, S. Xu, N. Glossop, A. Viswanathan, J. Borgert, H. Schulz and B. J. Wood, "Electromagnetic Track- ing for Thermal Ablation and Biopsy Guidance: Clinical Evaluation of Spatial Accuracy," Journal of Vascular and Interventional Radiology, Vol. 18, No. 9, 2007, pp. 11411150. doi:10.1016/j.jvir.2007.06.014

[15] L. Appelbaum, J. Sosna, Y. Nissenbaum, A. Benshtein, and S. N. Goldberg, "Electromagnetic Navigation System for CT-Guided Biopsy of Small Lesions," American Journal of Roentgenology, Vol. 196, No. 5, 2011, pp. 1194-1200. doi:10.2214/AJR.10.5151

[16] J. Hummel, M. Figl, C. Kollmann, H. Bergmannb and W. Birkfellner, "Evaluation of a Miniature Electromagnetic Position Tracker," Medical Physics, Vol. 29, No. 10, 2002, pp. 2205-2212. doi:10.1118/1.1508377 